\title{
EL CONFLICTO MUJER-EMBRIÓN EN DEBATE PARLAMENTARIO SOBRE EL ABORTO
}

\author{
SUSANA ROSTAGNOL \\ Universidad de la República, Uruguay
}

\begin{abstract}
Resumen: Este artículo analiza el conflicto mujer-embrión en los debates del Senado uruguayo entre octubre y noviembre 2007 en ocasión de la discusión del proyecto de ley que legaliza el aborto. Intenta dar cuenta de algunas nociones subyacentes a la discusión, a las que clasifica sintéticamente como aquellas que promueven el 'control patrimonial del cuerpo' y las que promueven la autonomía. Desde esta perspectiva se analiza el carácter abstracto o concreto del aborto en las distintas argumentaciones, para luego discutir el carácter de persona otorgado al zef (zigoto-embrión-feto) en base a fundamentos biomédicos. Luego se discute el carácter de persona moral asignado a la mujer embarazada. Finalmente se presenta el aborto como un hecho cuya significación hace a la organización de las relaciones sociales.
\end{abstract}

Palabras claves: aborto; persona; parlamento.

\section{Introducción}

En los debates parlamentarios llevados a cabo en Uruguay a fines del 2007 respecto al proyecto de Ley de Defensa del Derecho a la Salud Sexual y Reproductiva que permite el aborto por voluntad de la mujer hasta la semana 12, el conflicto de intereses mujer-embrión ocupó un lugar central. No es una discusión novedosa en si misma, sin embargo su actualidad hace que amerite un análisis. El debate llega a un punto muerto dado la inconmensurabilidad en tanto imposibilidad de diálogo al tratarse de principios no negociables en una y otra de las premisas. ' En el debate, hubo también otras argumentaciones basadas en la 'oportunidad política' de votar un proyecto de Ley que despenaliza el aborto. En este

Copyright $\odot 2008$ by Revista Estudos Feministas.

' Danielle Ardaillon denomina différends siguiendo a Lyotard, a estos "conflictos sin solución, son discusiones que no llegan a un consenso definitivo" (Daniele ARDAlLLON, 1997). 
artículo intentaré dar cuenta de algunos de los elementos involucrados y subyacentes en la discusión centrada en el conflicto mujer-embrión. ${ }^{2}$

\section{La Ideología del nombre}

Conocer es nombrar. No es necesario ser foucaultiano para reconocer que aquello que no forma parte del discurso no existe. Asimismo, al nombrar damos una determinada identidad a aquello que es nombrado. Por lo tanto cada perspectiva ideológica, al marcar una determinada manera de organizar el mundo, procurará los términos que le sean adecuados a sus fines. En el caso del aborto existe una batalla por el nombre del 'producto de la concepción': zigoto, embrión, feto, nonato, niño, bebé; dejando de lado la otra discusión también ligada al nombre: persona, ser humano, vida, persona en potencia, el por nacer, etc. En este artículo he optado por denominarlo de manera genérica zigotoembrión-feto (zef), no obstante reservé la denominación de embrión para el título. También existe una lucha por el nombre en cuanto a la mujer: mujer, madre, gestante.

Cada uno de los términos mencionados se relaciona con una manera específica de organizar y actuar en el mundo, implica una ideología diferente. Las propuestas en pugna pueden sintetizarse en aquellas que promueven un "control patrimonial del cuerpo" 3 y aquellas que promueven la autonomía de todos los individuos, hombres y mujeres.

\section{Los argumentos: entre lo abstracto y lo concreto}

Los argumentos esgrimidos por los legisladores contrarios a la despenalización del aborto generalmente se basan en fundamentos abstractos que refieren a una mujer que no se corresponde necesariamente con las mujeres concretas que abortan. Algunos de ellos recurren a doctrinas religiosas y/o metafísicas que aluden al momento en que el alma entra al zef, o al inicio de la vida en tanto persona.

Transcribo, a modo de ejemplo, algunos fragmentos tomados del diario de actas de las sesiones del Senado,

[...] consideramos que esas causales son extremadamente subjetivas ${ }^{4}$ pues la norma proyectada expresa: 'a su juicio'. Esta expresión [...] denota claramente la subjetividad que tiene el criterio, en el sentido de que la madre considere que a su juicio esas causales le impiden seguir con el embarazo. En esta materia está en juego el derecho a la vida, que, creemos, no es cualquier derecho, sino el primer escalón [...] ocupa un lugar en materia de valores superiores, por encima de los pragmatismos o realismos [...] el valor de la vida es un concepto demasiado caro e importante.

\footnotetext{
${ }^{2}$ Este análisis integra una investigación mayor sobre las prácticas y representaciones del aborto voluntario en Uruguay entre el 2003 y 2007. El material analizado en esta oportunidad comprende los diarios de sesiones del Senado correspondientes ( 16 y 17 de octubre y 6 de noviembre 2007) en que se trató el proyecto de ley de defensa de los derechos sexuales y reproductivos. El resto del material utilizado en el análisis proviene del trabajo de campo realizado en distintos centros de salud (nivel primario de atención y hospitales), entrevistas con personal de salud; con mujeres que abortaron y mujeres embarazadas que nunca consideraron abortar realizadas entre el 2004 y 2007.

${ }^{3}$ Giulia TAMAYO, 2001.

${ }^{4}$ Se refiere a las contenidas en el proyecto de ley, Art. 9 (condiciones): "Para ejercer el derecho reconocido en el artículo anterior, la mujer alegará ante el médico circunstancias derivadas de las condiciones en que ha sobrevenido la concepción, situaciones de penuria económica, sociales, familiares o etarias, que a su criterio le impidan continuar con el embarazo en curso".

${ }^{5}$ Senador Da Rosa, Diario de Sesiones, 39० Sesión Ordinaria, 16 oct. 2007.
} 
En otra intervención, el mismo senador afirmó "En base a una meditada decisión, consideramos que la jerarquía del derecho a la vida está por encima de otras consideraciones $[\ldots] " .6$

Otras argumentaciones han privilegiado lo que denominan un 'beneficio para la sociedad' como elemento fundamental:

Todos los que hemos asumido posiciones sobre este tema, lo hemos hecho considerando que es lo mejor para la sociedad [...] Tenemos una población casi estable, que crece muy poco [...] la baja tasa de natalidad y la emigración, dos indicadores que vaticinan un futuro oscuro y problemático para la sociedad uruguaya.

Esta argumentación coloca el énfasis en la tensión individuo-sociedad. El liberalismo utilitario propone que a mayor bienestar individual, mayor bienestar colectivo, esto supone una "sociedad orgánica-simbiótica, en la cual las pérdidas individuales se compensan con las ganancias sociales". ${ }^{8}$ Esta postura es contraria a la expresa en el fragmento transcripto. El legislador señala la necesidad de aumentar el número de nacimientos - por lo tanto las mujeres no deben abortar aún cuando no quieran ser madres - porque la sociedad lo necesita, pero nada dice de quien debe ser responsable por el bienestar de esos niños. De modo que la pregunta central es ¿Quién debe hacerse cargo de una nueva criatura, y cuáles son sus derechos y deberes respecto a ella? Si se considera que una mujer es un sujeto moral, es decir capaz de tomar sus propias decisiones, entonces debe tener la posibilidad de asumir responsabilidades y compromisos. En tanto solo las mujeres tienen la posibilidad de embarazarse, entonces son ellas quienes deben asumir el compromiso de llevar o no a término su embarazo, y comparar esa opción con los otros compromisos y responsabilidades que ya tenga asumidos. ${ }^{9}$ Este razonamiento empata con las nociones de libertad y libre opción propias del liberalismo.

Sin embargo, si la mujer está inhabilitada de elegir - tal como en el fragmento transcripto - no parece sensato exigirle deberes de cuidados hacia un hijo que eligió no tener, sino que el Estado la obligó a llevar a término su embarazo. Entonces, ¿a quién le corresponde asumir los deberes para con ese ser? Marta Lamas señala que "hoy la cuestión crucial sobre el aborto radica en determinar quién decide si nacen o no ciertas criaturas". ${ }^{10}$ ¿A quién le compete decidir la continuación o la interrupción de un embarazo?

El mandato social no censura tanto la interrupción del embarazo sino que sea la mujer quien lo decida, porque no son sujetos legitimados socialmente para tomar la decisión de interrumpir su propio embarazo. El cuerpo, la sexualidad de la mujer son tratados como asunto público $[\ldots]$.

\footnotetext{
${ }^{6}$ Senador Da Rosa, Partido Nacional, miembro informante de la Comisión de Salud Pública del Senado. El Partido Nacional es conservador, se ha mantenido tradicionalmente en contra de la legalización del aborto. Existía un mandato del Partido de votar en contra del proyecto de ley que legalizaba el aborto (Diario de Sesiones, $39^{\circ}$ Sesión Ordinaria, 16 oct. 2007).

${ }^{7}$ Senador Amaro, Partido Colorado. En relación al aborto, el Partido Colorado tradicionalmente ha adoptado medidas liberales, no obstante en el gobierno anterior, el presidente Dr. Jorge Batlle, del Partido Colorado, había manifestado su intención de vetar una ley que legalizara la práctica del aborto. En esta legislatura los senadores estaban en libertad de acción (Diario de Sesiones, $39^{\circ}$ Sesión Ordinaria, 16 oct. 2007).

8 Josefina FIGUEIRA-MCDONOUGH, 1990, p. 29, traducción de la autora.

9 FIGUEIRA-MCDONOUGH, 1990.

${ }^{10}$ Marta LAMAS, 2004, p. 35.

$"$ Susana ROSTAGNOL, 2006, p. 67.
} 
Las argumentaciones de los legisladores favorables a la legalización del aborto se apartan de las abstracciones y generalizaciones, de modo que la singularidad de la mujer concreta que aborta es observada exclusivamente en algunos discursos a favor de la aprobación del proyecto de Ley.

Ellas están solas [...] quien debe someterse a un aborto es la mujer. [...] Estamos, señor Presidente, nos guste o no, administrando un capital ajeno. Podemos disfrazar ese hecho de mil maneras distintas; podemos manejar [...] los porcentajes de mortalidad materna como consecuencia de abortos realizados en condiciones inapropiadas o hablar [...] del interés ético de la sociedad en preservar el valor de la vida humana por encima de cualquier consideración [...] estamos nosotros, los legisladores hombres, decidiendo en abstracto sobre lo que es en concreto una realidad a la que solamente, y solas, se deben enfrentar las mujeres.

Aquí es prácticamente la única oportunidad donde parece aludirse al hecho que la mujer no eligió quedar embarazada, está forzada por ser mujer, "esa falta de libertad original", al decir de Laura Klein. ${ }^{13}$

Otros legisladores en sus intervenciones se refirieron a la necesidad de respetar la pluralidad de posturas, sin imponer una sobre otras, lo cual se aleja de los valores absolutos y generalizados observados en las transcripciones anteriores. También aparecen alusiones a la necesidad de incorporar una perspectiva de género al tema.

Consideramos que va a ser difícil que se incorpore la perspectiva de género en las políticas de salud, si el concepto que se utiliza es sólo y estrictamente biomédico y si en los diagnósticos de situación del país no están presentes las relaciones sociales de poder entre mujeres y hombres, de las que derivan las situaciones de violencia que sufre la mujer $[\ldots .$.$] cuando decide interrumpir su embarazo, ya que es penalizado [\ldots]^{14}$

Es interesante la forma en que la senadora asocia los fundamentos biomédicos con el mantenimiento de las relaciones de inequidad de género, colocándolos al servicio de la postura que he denominado de 'control patrimonial del cuerpo'.

Esta asociación se ve reforzada en la intervención de un legislador contrario a la despenalización del aborto quien se expresaba:

[...] desde nuestra óptica, existe vida desde la concepción. A medida que han ido pasando los años, la evidencia científica avala cada vez más esa posición [...] Está científicamente comprobado y demostrado que con la unión de los 23 cromosomas paternos y los 23 cromosomas maternos, producida en el acto de penetración del óvulo por el espermatozoide, o sea en la fecundación, se genera un individuo único e irrepetible [...] El embrión es 'otro' con relación a la madre - está en la madre, aunque podría no estarlo, pero no es de ella. Y más adelante, el mismo legislador cita a Juan Pablo II: La vida es un bien absoluto y no relativo. ${ }^{15}$

\footnotetext{
${ }^{12}$ Senador Breccia, Frente Amplio. La mayoría de los legisladores del Frente Amplio han manifestado su acuerdo con la legalización del aborto. Es especialmente significativo que el presidente Dr. Tabaré Vázquez haya advertido en numerosas ocasiones, su intención de vetar cualquier ley que despenalice el aborto. Esto le da al tema un matiz político nada despreciable, ya que algunos legisladores mencionan su desacuerdo en tratar un tema que los enfrentaría al presidente, lo cual revela una severa limitación a la independencia del Poder Legislativo respecto al Ejecutivo (Diario de Sesiones, $39^{\circ}$ Sesión Ordinaria, 16 oct. 2007).

${ }^{13}$ Laura KLEIN, 2005, p. 45.

${ }^{14}$ Senadora López, Frente Amplio. Diario de Sesiones, 39 Sesión Ordinaria, 16 oct. 2007.

${ }^{15}$ Senador Long, Partido Nacional. Diario de Sesiones, $39^{\circ}$ Sesión Ordinaria, 16 oct. 2007.
} 
Este fragmento une los fundamentos de la biomedicina con los de la religión católica, específicamente a partir de los discursos que emanan del Vaticano. Esto es un punto a subrayar, la ciencia y el catolicismo se unen para fortalecer una postura que dificulta a las mujeres actuar como sujetos morales.

En un punto nodal en el conflicto mujer-zef está la definición de persona.

\section{¿Quién es/Qué es una persona?}

Si consideramos que el zef es una persona en potencia, entonces su destrucción es prima facie moralmente inadmisible de acuerdo a los planteos de John Noonan entre otros; ${ }^{16}$ quienes comparten la idea que indiferentemente si el status es de persona o persona en potencia, el zigoto ya posee todos los derechos inherentes al ser humano, siendo el primero de ellos a la vida, en realidad debería decirse a nacer. Esta es la idea impulsada por algunos legisladores contrarios a la despenalización. Frente a estas argumentaciones, Paul Bassen responde que "[...] en ningún lado fuera del debate sobre el aborto, existe un precedente donde un prospecto futuro pueda crear un bien presente". ${ }^{17}$ Desde otro lugar, la argumentación de Thomson ${ }^{18}$ en defensa del aborto, aún cuando pudiese considerarse que el feto fuese una persona, radica en proponer que nada obliga a la mujer a sacrificar su cuerpo para gestar durante nueve meses. Este argumento ha levantado discrepancias.

Si bien los contrarios al aborto lo consideran un homicidio, Laura Klein advierte que el aborto no refiere a la finalización de la vida, sino a la interrupción de un embarazo, colocando en el embarazo el centro de la cuestión. Sin embargo, una y otra vez los argumentos - especialmente aquellos contrarios a la legalización del aborto, enfatizan la noción de vida (a la que le adjudican el carácter de persona) y en ello basan su posición.

Nadie argumenta en contra de la existencia de vida (en el zigoto, embrión y feto), la noción que se discute es si puede considerarse vida 'humana', y más aún, si puede considerarse 'persona'.

En los últimos años, han sido los argumentos biomédicos los que intentan sentar las bases del comienzo de la existencia como persona del zef. Si bien tradicionalmente se trataba de un argumento moral, del cual el derecho - en tanto disciplina - y la filosofía daban cuenta; ahora es el biomédico el discurso más legitimado. Sin embargo, se mantienen algunos cuestionamientos. Existen pocos desacuerdos sobre la perspectiva biológica de individualidad en desarrollo, pero existen grandes desacuerdos en relación a la etapa en la cual las formas particulares de vida humana son titulares de cualquiera sean los derechos que una comunidad garantiza para sus miembros. Son los Códigos Civiles de los distintos países los que en definitiva definen las características necesarias para acceder a la titularidad de derechos, y esto depende de la definición de persona. Los argumentos acerca de las reacciones biológicas del feto y los logros científicos que hacen que un feto sea viable a tempranas etapas no clarifica este asunto, el resultado depende de una definición normativa, el atributo de un status específico. ${ }^{19}$

Tanto la evidencia histórica como antropológica muestran la inexistencia de un criterio universal para caracterizar a la 'persona'. Li Puma ${ }^{20}$ advierte que en todas las culturas,

\footnotetext{
${ }^{16}$ Este tema está ampliamente tratado en: Bertha ALVAREZ MANNINEN, 2007.

17 Paul BASSEN, 1982, p. 333.

18 Judith Jarvis THOMSOM, 1971.

19 FIGUEIRA-MCDONOUGH, 1990, p. 32.

${ }^{20}$ Edward LI PUMA, 1998.
} 
la 'persona' [personhood] posee aspectos o modalidades individuales (con atributos internos, constituyentes de una sustancia) y aspectos o modalidades dividuales (existentes a partir de la relación con otros). La tradición occidental hegemónica considera a la persona en términos de atributos internos completamente individualizados y definidos. Desde esta perspectiva es que se considera al zef como una persona. Sin embargo, si consideramos la modalidad dividual podría no serlo. Las mujeres embarazadas que deciden abortar no consideran el zef dentro del 'mapa relacional' (no tiene un lugar en el espacio social de esa mujer), contrariamente con aquellas que sí quieren continuar su embarazo. ${ }^{21}$ Esto significa que para las mujeres embarazadas, el zef es una persona en tanto integrado en una red de relaciones sociales, siendo la primera de ella la relación madre-hijo; cuando no lo perciben dentro de esta red, no es considerado persona, por lo tanto al abortar no 'matan' a nadie (porque no existe nadie), sino que interrumpen un embarazo. Estos aspectos no fueron considerados en las discusiones parlamentarias. Como mencioné, los argumentos se centraron en la biomedicina y la religión.

\section{El criterio de persona en el debate sobre el aborto}

Entre aquellos para quienes el zef es una persona, existe un grupo no despreciable de individuos que considera que el aborto debería permitirse bajo ciertas circunstancias, tales como si el embarazo es resultado de una violación, si el embarazo pone en riesgo la vida de la mujer; si hay incompatibilidad con la vida extrauterina. Josefina FigueiraMcDonough advierte la incongruencia entre ambos argumentos:

el derecho fundamental a la vida del feto qua persona no puede ser la consecuencia de la forma en que se concibió. El carácter de persona del feto es, por definición, intrínseco y contingente a las causas del embarazo: un acto de amor, explotación, 0 violencia. $^{2 /}$

\section{LAS RESPUESTAS BIOMÉDICAS}

La tecnología - en especial el ultrasonido - pasó a ocupar un lugar central en la construcción social del zef como persona. Esto se relaciona con dos aspectos generales de nuestra cultura de comienzos del siglo XXI, por una parte la popularización de los medios audiovisuales como modo de relacionarse con el mundo (aprehenderlo, entenderlo y explicarlo) y por otro con la creciente 'espectacularización' de la vida cotidiana, es decir la vida como espectáculo, puesto de manifiesto en los abundantes talk shows, programas del tipo Gran hermano, a modo de ejemplos. Finalmente, existe un tercer aspecto que coloca al ultrasonido en un lugar relevante: refuerza el status de autoridad científica, el médico 'observa' al zigoto-embrión-feto 'directamente', 'sin intermediación' de la mujer-madre (la intermediación tecnológica no cuenta, actuando como 'extensión' de los sentidos del médico ecografista). La tecnología del ultrasonido le permite relacionarse a través del ecógrafo, prescindiendo de la madre. Permite al médico tratar al zef como si fuese autónomo, como un paciente.

Como contraparte, frecuentemente las mujeres embarazadas son tratadas como contenedor del verdadero paciente: el zef.

La creciente tendencia a conceptuar los derechos del feto como separados y opuestos a los derechos de las mujeres legitima una seria distorsión - literalmente una descorporización

\footnotetext{
${ }^{21}$ Aspecto tratado en Rafael SANSEVIERO, 2003, y que coincide con la información proveniente de las entrevistas realizadas en mi propia investigación.

22 FIGUEIRA-MCDONOUGH, 1990, p. 11, traducción de la autora.
} 
- de la condición de embarazo. Esta forma de conceptuar (de nombrar y de definir la situación de embarazo), perpetúa la seria erosión de los derechos de las mujeres a la autonomía reproductiva, ${ }^{23}$ reforzando el 'control patrimonial del cuerpo'.

La conversión de un examen de rutina en un espectáculo ${ }^{24}$ es llevada adelante tanto por el/la ecografista como por la mujer y sus acompañantes, retroalimentándose mutuamente. El espectáculo anticipa la existencia social del zef, "en lo que podría ser descripto como un proceso de inclusión social equivalente a una couvade urbana, contemporánea, mediada por la tecnología de las imagenes", ${ }^{25}$ se lleva a cabo una metamorfosis de imagen fragmentada del zef en bebé real en la pantalla. Lilian Chazan afirma que "la transformación del examen en espectáculo y objeto de consumo es fundamental dentro del proceso de construcción del feto como Persona, en la medida en que le confiere visibilidad en una cultura en la cual lo visual es preponderante". ${ }^{26} \mathrm{El}$ proceso de subjetivación del zef es realizado tanto por el ecografista como por la madre y acompañantes que acuden al examen. Se le atribuye intencionalidad a su actividad intrauterina ('duerme', 'se despereza'), y a sus estados de ánimo ('mirá que feliz está'), se lo caracteriza como si fuese un bebé con comentarios tales como 'está saludando'. En otras palabras, se le atribuye al zef una subjetividad propia de los seres humanos titulares de derechos.

\section{El status de persona en la mujer embarazada}

¿La mujer es una persona?, desde la perspectiva del "control patrimonial de los cuerpos" obviamente no lo es, aunque no se lo especifica en los discursos en los cuales subyace esta perspectiva ideológica. Laura Klein ${ }^{27}$ es muy aguda al mostrar la equivalencia entre el aborto en el relato bíblico y en el relato contemporáneo. En el primero el damnificado era el padre, y ahora el hijo. Las mujeres siguen quedando excluidas de la titularidad de derechos. Los discursos provenientes de los contrarios a la legalización del aborto describen a estas mujeres como asesinas, carentes de principios morales, irresponsables y profundamente egoístas.

Mientras se prive a las mujeres de la autodeterminación en materia reproductiva - es decir mientras no se le dé el status de sujeto mora l - se las mantiene en un lugar socialmente subordinado.

Por ello el aborto es bastante más que un asunto de mujeres, aunque es principalmente un asunto de mujeres que acontece en el cuerpo de una determinada mujer. El aborto forma parte de la gran política, de las formas en que las sociedades se dan para organizar la convivencia y para construir las relaciones sociales, especialmente las relaciones de género. Las discusiones parlamentarias en torno al proyecto de Ley de Defensa del Derecho a la Salud Sexual y Reproductiva refieren a la organización social y sus jerarquías, el control patrimonial del cuerpo o a la autonomía de las personas.

\footnotetext{
${ }^{23}$ Kelly MAIER, 1989, p. 19.

${ }^{24}$ Este aspecto del ultrasonido está trabajado en: Lilian CHAZAN, 2005.

${ }^{25}$ CHAZAN, 2005, p. 8.

${ }^{26}$ CHAZAN, 2005, p. 13.

27 KLEIN, 2005.
} 


\section{Referencias bibliográficas}

ALVAREZ MANNINEN, Bertha. "Revisiting the Argument from Fetal Potencial". Philosophy, Ethics, and Humanities in Medicine, n. 2, p. 7, 2007. Disponible en: http://www.peh-med.com/ content/2/1/7. Visitado en: feb. 2008.

ARDAILLON, Daniele. Cidadanía de corpo enteiro: discursos sobre o aborto em número e gênero. 1997. Tese (Doutorado em Sociología) - Departamento de Sociología, Faculdade de Filosofia, Letras e Ciencias Humanas, USP, São Paulo, 1997.

BASSEN, Paul. "Present Stakes and Future Prospects: The Status of Early Abortions". Philosophy and Public Affairs, Reino Unido, v. 11, n. 4, p. 314-337, 1982.

CHAZAN, Lilian Krakowski. "'O melhor filmed a miha vida!' O ultra-som, o corpo grávido e o corpo fetal". In: VI REUNIÓN DE ANTROPOLOGÍA DEL MERCOSUR, nov. 2005, Montevideo.

FIGUEIRA-MCDONOUGH, Josefina. "Abortion, Ambiguous Criteria and Confusing Policies". Affilia, Washington, v. 5, n. 4, p. 27-54, invierno 1990.

KLEIN, Laura. Fornicar y matar. El problema del aborto. Buenos Aires: Planeta. 2005.

LAMAS, Marta. "Aborto: viejo problema y nuevos dilemas". Perinatalogia y Reproducción Humana, México, n. 18, p. 34-43, 2004.

LI PUMA, Edward. "Modernity and Forms of Personhood in Melanesia". In: LAMBEK, Michael; STRATHERN, Andrew. Bodies and Persons. Comparative Perspectives from Africa and Melanesia. Reino Unido: Cambridge University Press, 1998. p. 53-79.

MAIER, Kelly. "Pregnant Women: Fetal Containers or People with Rights?". Affilia, Washington, v. 4, n. 2, p. 8-20, verano 1989.

ROSTAGNOL, Susana. "Territorio femenino, discurso masculine". In: RED DE SALUD DE LAS MUJERES LATINOAMERICANAS Y DEL CARIBE. Tu cuerpo, tu vida, tu decides. Santiago de Chile, 2006. (Publicado por primera vez en 2001).

SANSEVIERO, Rafael. Condena, tolerancia, negación. El aborto en Uruguay. Montevideo: CIIIP-UPAZ, 2003.

TAMAYO, Giulia. Bajo la piel. Lima: Flora Tristán, 2001.

THOMSON, Judith Jarvis. "A Defense of Abortion". Philosophy and Public Affairs, New Jersey, v. 1, n. 1, p. 47-66, 1971.

[Recebido em maio de 2008 e aceito para publicação em junho de 2008]

\section{The Woman-Embryo Conflict in the Abortion Debate at the Parliament}

Abstract: This article analyzes the woman-embryo conflict discussed by the Uruguayan Senate during the debates on abortion legalization in October and November 2007. Its aim is to show the underlying notions, which are classified as those that promote the 'patrimonial control over the body' and those that promote autonomy. From that perspective, it analyzes the abstract or concrete standpoint given to abortion in the Senators arguments. Then, it discusses the personhood assigned to zef (zygote-embryo-fetus) based on biomedical arguments. It also discusses the attribute of moral person given/or not to the pregnant woman. Finally it shows that abortion is basically a fact that influences directly in the organization of social relations.

Key Words: Abortion; Personhood; Parliament. 